\title{
A FRACTAL PLANCHEREL THEOREM
}

\author{
URSULA M. MOLTER AND LEANDRO ZUBERMAN
}

\begin{abstract}
A measure $\mu$ on $\mathbb{R}^{n}$ is called locally and uniformly $h$-dimensional if $\mu\left(B_{r}(x)\right) \leq h(r)$ for all $x \in \mathbb{R}^{n}$ and for all $0<r<1$, where $h$ is a real valued function. If $f \in L^{2}(\mu)$ and $\mathcal{F}_{\mu} f$ denotes its Fourier transform with respect to $\mu$, it is not true (in general) that $\mathcal{F}_{\mu} f \in L^{2}$ (e.g. [10]).

However, in this paper we prove that, under certain hypothesis on $h$, for any $f \in L^{2}(\mu)$ the $L^{2}$-norm of its Fourier transform restricted to a ball of radius $r$ has the same order of growth than $r^{n} h\left(r^{-1}\right)$, when $r \rightarrow \infty$. Moreover, we prove that the ratio between these quantities is controlled by the $L^{2}(\mu)$-norm of $f$ (Theorem 3.2). By imposing certain restrictions on the measure $\mu$, we can also obtain a lower bound for this ratio (Theorem 4.3).

These results generalize the ones obtained by Strichartz in [10] where he considered the particular case in which $h(x)=x^{\alpha}$.
\end{abstract}

\section{INTRODUCTION}

We will say that a measure $\mu$ is locally and uniformly $h$-dimensional (or shortly $\mu$ is an $h$-dimensional measure) if and only if, the following condition holds

$$
\mu\left(B_{r}(x)\right) \leq h(r) \quad \forall x \in \mathbb{R}^{n}, \forall 0<r<1,
$$

where $B_{r}(x)$ is, as usual the ball of radius $r$ centered at $x$. We consider functions $h:[0,+\infty] \rightarrow \mathbb{R}$ that are non-decreasing, continuous and such that $h(0)=0$. We further require $h$ to be doubling, i. e. there exists a constant $c>0$ such that $h(2 x)<\operatorname{ch}(x)$. Such a function will be called dimension function. A particular example is $h(x)=x^{\alpha}$, which was analyzed by Strichartz in [10]. In that case we will say indistinctly that $\mu$ is $h$-dimensional or that $\mu$ is $\alpha$-dimensional.

Allowing $h$ to be more general has already proven to be useful (see for example $[6],[5],[2])$ and it enables us to obtain a lower bound on measures which were not included in previous results (see Section 5).

If $\mu$ is locally and uniformly 0 -dimensional, meaning that the measure of any ball of radius one is bounded, then each $f \in L^{2}(\mu)$ defines a tempered distribution, mapping each test function $\varphi$ in the Schwartz space $\mathscr{S}$ into $\int f \varphi d \mu$. Therefore, its Fourier transform is also a tempered distribution defined by

$$
\varphi \mapsto \int \hat{\varphi} f d \mu \quad \varphi \in \mathscr{S}
$$

Date: April 8, 2008.

1991 Mathematics Subject Classification. 42B10(28A80).

Key words and phrases. Hausdorff Measures, Fourier Transform, Dimension, Plancherel.

The research of the authors is partially supported by Grants: CONICET PIP 5650/05, UBACyT X108, and PICT-03 15033. 
where $\hat{\varphi}$ is the usual Lebesgue Fourier transform. We will denote by $\mathcal{F}_{\mu} f$ this 'distributional' Fourier transform of an $f \in L^{2}(\mu)$. If $f \in L^{1}(\mu) \cap L^{2}(\mu)$ then it is easy to see that $\mathcal{F}_{\mu} f(\xi)=\int f(x) e^{i \xi x} d \mu(x)$, see for example [1].

Strichartz proved ([10]) that if $f \in L^{2}(\mu)$ and $\mu$ is zero dimensional then $\mathcal{F}_{\mu} f$ belongs to $L^{2}\left(e^{-t|\xi|^{2}}\right)$ for any $t>0$ and therefore to $L_{l o c}^{2}$. Note that if $h$ is one of our dimension functions, we have inmediatly that $\mu$ is 0 -dimensional.

In this paper, our goal is to prove for any $h$-dimensional measure $\mu$, an analogue to Plancherel's Theorem in $L^{2}\left(\mathbb{R}^{n}\right)$ with the Lebesgue measure. In fact we are going to show existence of upper and lower bounds for the ratio between $r^{n} h\left(r^{-1}\right)$ and the norm of the Fourier transform in $L^{2}(\mu)$ restricted to the ball of radius $r$. The hypothesis under which we obtain the existence of the upper bound are more general than the ones we need for the existence of the lower ones.

The $h$-dimensional Hausdorff measure is defined as (see for example [6]):

$$
\mathcal{H}^{h}(E)=\lim _{\delta \rightarrow 0}\left(\inf \left\{\sum_{i=1}^{\infty} h\left(\left|U_{i}\right|\right): E \subset \bigcup_{i \geq 1} U_{i} \text { and }\left|U_{i}\right| \leq \delta\right\}\right),
$$

and $\mathcal{H}_{\llcorner E}^{h}$ will denote its restriction to a set $E$.

The $h$-lower density of a set $E$ in $x$ is (see for example [3]):

$$
\underline{D}\left(\mathcal{H}^{h}\left\llcorner_{E}, x\right)=\liminf _{r \rightarrow 0} \frac{\mathcal{H}^{h}\left(E \cap B_{r}(x)\right)}{h(2 r)} .\right.
$$

The upper density is defined by taking limsup in the above equation. We will introduce one aditional definition.

\section{Definition 1.1.}

- A set $E$ will be said to be an $h$-regular set if both, upper and lower densities, are equal to one in $\mathcal{H}^{h}$ almost every point of $E$. In symbols,

$$
\underline{D}\left(\mathcal{H}^{h}{ }_{\llcorner E}, x\right)=\bar{D}\left(\mathcal{H}^{h_{\llcorner}}, x\right)=1,
$$

for $\mathcal{H}^{h}$ almost every point of $E$.

- If the lower density is greater than a positive constant for $\mathcal{H}^{h}$-almost every point of $E$ we will say that $E$ is an h-quasi regular set.

The lower bound that we obtain (see Theorem 4.2) will be stated for the measure $\mathcal{H}^{h}$ restricted to an $h$-dimensional and quasi regular set. In section 5 we will show an example of a set $E$ and a function $h$ such that $\mathcal{H}^{h}\left\llcorner_{E}\right.$ is $h$ - dimensional and $E$ is quasi regular. Additionally, we will prove that there does not exist any $\alpha$ such that $\mathcal{H}^{\alpha}\left\llcorner_{E}\right.$ is $x^{\alpha}$-dimensional and $E$ is quasi regular simultaneously. This example satisfies the hypothesis of our theorem 4.1 but does not satisfy the hypothesis of the analogous theorem 5.5 in [10].

\section{Some technical RESUlts}

Any $h$ dimensional measure $\mu$ is, in particular, locally finite, which means that for $\mu$ almost every $x$ there exists an $r>0$ such that $0<\mu\left(B_{r}(x)\right)<\infty$. Therefore, as Strichartz proved in [10], the strong $(p, p)$ estimate (for $p>1$ ) and the weak $(1,1)$ estimate hold for the maximal operator, defined for each $f \in L_{l o c}^{1}(\mu)$ as follows:

$$
M_{\mu} f(x)=\sup _{r>0} \frac{1}{\mu\left(B_{r}(x)\right)} \int_{B_{r}(x)}|f| d \mu .
$$


Precisely, we have the following theorem:

Theorem 2.1. Let $\mu$ be a locally finite measure on $\mathbb{R}^{n}$. For each locally integrable function $f$ we have:

(1)

$$
\mu\left(\left\{x: M_{\mu} f(x)>s\right\}\right) \leq \frac{c_{n}}{s}\|f\|_{1} \quad \forall f \in L^{1}(\mu) .
$$

(2) For $1<p \leq \infty$,

$$
\left\|M_{\mu} f\right\|_{p} \leq c_{p}\|f\|_{p} \quad \forall f \in L^{p}(\mu) .
$$

This theorem has many consequences which will be useful for our work. In particular, we have the following two corollaries:

Corollary 2.2. Let $f \in L^{1}(\mu)$. For $\mu$-almost every $x$,

$$
\lim _{r \rightarrow 0} \frac{1}{\mu\left(B_{r}(x)\right)} \int_{B_{r}(x)} f d \mu=f(x) .
$$

Corollary 2.3. Let $E$ be a h-regular set and let $f \in L^{1}(\mu)$. For $\mathcal{H}^{h}$-almost every $y \in E$ and for all $\varepsilon>0$ there exists $\delta>0$ such that

$$
\left|\int_{B_{r}(y)} f d \mu-h(r) f(y)\right| \leq \varepsilon h(r) \quad \forall r \leq \delta .
$$

The proofs of the Theorem and these Corollaries are straightforward applications of Besicovitch's covering Theorem and can be found in [10].

We also need the following quite technical Lemma, which will allow us to bound the ratio between $h$ and its dilation by $r(h(r t) / h(t))$ by a function in the weighted space $L^{1}\left(e^{-c r^{2}}\right)$.

Lemma 2.4. Let $h:[0, \infty) \rightarrow \mathbb{R}$ be a continuous, non-decreasing and doubling function $\left(h(2 x) \leq c_{d} h(x)\right)$. Then there exists a constant $\kappa>0$ such that

$$
h(r t) \leq c_{d} h(t) \max \left\{1, r^{\kappa}\right\} \quad \forall r, t>0 .
$$

Proof. First, note that $c_{d} \geq 1$, since in fact, the doubling condition can be restated as $c_{d} \geq h(2 x) / h(x)$ and this quantity is not smaller than 1 because $h$ is a non decreasing function.

If $r<1$, since $h$ is non-increasing, we have $h(r t) \leq h(t)$. If $r \geq 1$ we choose the only non negative integer $k$ such that $2^{k-1}<r \leq 2^{k}$. So, $h(r t) \leq h\left(2^{k} t\right) \leq c_{d}^{k} h(t)$. Observe that $k$ was chosen such that $k \leq \frac{\log r}{\log 2}+1$, and therefore it then follows that $c_{d}^{k} \leq c_{d} \cdot r^{\log c_{d} / \log 2}$. The proof is complete by taking $\kappa=\log c_{d} / \log 2$.

Recall that we are dealing with $h$-dimensional measures which means that the measure of the balls of radius $r<1$ is bounded. The next lemma provides a control of the measure of the "big" balls, ie, those balls of radius greater than one, for which the estimate (1.1) does not hold.

Lemma 2.5. Let $\mu$ be a locally h-dimensional measure on $\mathbb{R}^{n}$. If $r>1$, then $\mu\left(B_{r}(x)\right) \leq C r^{n}$, for some $C$ independent of $x$.

Proof. Denote by $Q$ the minimal cube centered at $x$ that contains the ball $B_{r}(x)$ :

$$
Q=Q(x, r)=\left\{y \in \mathbb{R}^{n}:\|x-y\|_{\infty}<r\right\} \supset B_{r}(x) .
$$


Let $k$ be the (unique) integer such that $k-1<r \sqrt{n} \leq k$. $Q$ can be divided into $k^{n}$ smaller cubes of half-side $\frac{r}{k}$. Each of these cubes is contained in a ball of radius $r_{0}=\sqrt{n} \frac{r}{k} \leq 1$. So, we obtain:

$$
\mu\left(B_{r}(x)\right) \leq \mu(Q) \leq k^{n} \mu\left(B_{r_{0}}\left(x^{\prime}\right)\right) \leq k^{n} h\left(\frac{\sqrt{n} r}{k}\right) .
$$

Since $\sqrt{n} \frac{r}{k} \leq 1$, it follows that $h\left(\sqrt{n} \frac{r}{k}\right) \leq h(1)$. On the other hand, by the choice of $k, k^{n}<(r \sqrt{n}+1)^{n} \leq r^{n}(\sqrt{n}+1)^{n}$, and we obtain,

$$
\mu\left(B_{r}(x)\right) \leq(\sqrt{n}+1)^{n} h(1) r^{n} .
$$

\section{UPPER BOUNDS}

Our first result is an upper estimate for the $L^{2}$-norm of the Fourier transform of a function $f \in L^{2}(\mu)$.

Theorem 3.1. Let $\mu$ be a locally and uniform $h$-dimensional measure, where $h$ is a dimension function. Suppose that $h$ defines a dimension not greater than $n$ in the sense that $\lim _{t \rightarrow 0} t^{n} / h(t)=0$. Then,

$$
\sup _{0 \leq t \leq 1} \frac{\sqrt{t^{n}}}{h(\sqrt{t})} \int e^{-t|\xi|^{2}}\left|\mathcal{F}_{\mu} f(\xi)\right|^{2} d \xi \leq c\|f\|_{2}^{2}:=c \int|f|^{2} d \mu \quad \forall f \in L^{2}(\mu) .
$$

Proof.

First Step We will prove that:

$$
\sqrt{t^{n}} \int\left|\mathcal{F}_{\mu} f(\xi)\right|^{2} e^{-t|\xi|^{2}} d \xi=\pi^{n / 2} \iint e^{-|x-y|^{2} / 4 t} f(x) \overline{f(y)} d \mu(x) d \mu(y) .
$$

Remembering the inverse Fourier transform for the gaussian function,

$$
\int e^{-t|\xi|^{2}} e^{i x \xi} d \xi=\sqrt{t^{-n}} \pi^{n / 2} e^{-|x|^{2} / 4 t}
$$

if $f$ is integrable then equation (3.2) follows from Fubini's theorem, since:

$$
\begin{aligned}
\sqrt{t^{n}} \int\left|\mathcal{F}_{\mu} f(\xi)\right|^{2} e^{-t|\xi|^{2}} d \xi & =\sqrt{t^{n}} \iiint f(x) \overline{f(y)} e^{i(x-y) \cdot \xi} e^{-t|\xi|^{2}} d \mu(x) d \mu(y) d \xi \\
& =\pi^{n / 2} \iint e^{-|x-y|^{2} / 4 t} f(x) \overline{f(y)} d \mu(x) d \mu(y) .
\end{aligned}
$$

Now, consider any $f \in L^{2}(\mu)$, not necessarily integrable. Let us define

$$
f_{k}(x)=f(x) \chi_{\{|x| \leq k\}}(x) \chi_{\{|f(x)| \leq k\}}(x) .
$$

This sequence converges to $f$ in $L^{2}(\mu)$. Also, since each $f_{k}$ is in $L^{1}(\mu)$, it satisfies (3.3). Using Beppo Levi's theorem, we have:

$$
\iint e^{-|x-y|^{2} / 4 t} f_{k}(x) \overline{f_{k}(y)} d \mu(x) d \mu(y) \rightarrow \iint e^{-|x-y|^{2} / 4 t} f(x) \overline{f(y)} d \mu(x) d \mu(y) .
$$

Since $f \in L^{2}(\mu)$, it follows that $\mathcal{F}_{\mu} f \in L^{2}\left(e^{-t|\xi|^{2}} d \xi\right)$. Hence we can apply the dominated convergence theorem, and

$$
\sqrt{t^{n}} \int\left|\mathcal{F}_{\mu} f_{k}(\xi)\right|^{2} e^{-t|\xi|^{2}} d \xi \rightarrow \sqrt{t^{n}} \int\left|\mathcal{F}_{\mu} f(\xi)\right|^{2} e^{-t|\xi|^{2}} d \xi
$$

which yields 3.2 . 
Second step

We will prove that, for any $y \in \mathbb{R}^{n}$ and $f \in L^{2}(\mu)$

$$
\frac{1}{h(\sqrt{t})} \int e^{-|x-y|^{2} / 4 t} f(x) d \mu(x) \leq C M_{\mu} f(y) .
$$

Using Fubini, on the left hand side of the inequality, we have that:

$$
\int e^{-|x-y|^{2} / 4 t} f(x) d \mu(x)=\int_{0}^{\infty} \frac{r}{2 t} e^{-r^{2} / 4 t} \int_{B_{r}(y)} f(x) d \mu(x) .
$$

Since

$$
\int_{B_{r}(y)} f(x) d \mu(x) \leq \mu\left(B_{r}(y)\right) M_{\mu} f(y)
$$

it follows that

$$
\int e^{-|x-y|^{2} / 4 t} f(x) d \mu(x) \leq M_{\mu}(y) \int_{0}^{\infty} e^{-r^{2} / 4 t} \frac{r}{2 t} \mu\left(B_{r}(y)\right) d r .
$$

We need to prove that the last integral is finite. To establish that, we split the integral into two parts, the first for $r<1$ (where (1.1) is valid) and the second for $r \geq 1$ (where lemma 2.5 can be applied). For $r<1$ we use the hypothesis to obtain:

$$
\begin{aligned}
\int_{0}^{1} e^{-r^{2} / 4 t} \frac{r}{2 t} \mu\left(B_{r}(y)\right) d r & \leq \int_{0}^{1} e^{-r^{2} / 4 t} \frac{r}{2 t} h(r) d r \\
& =\frac{1}{2} \int_{0}^{1 / \sqrt{t}} e^{-r^{2} / 4} r h(r \sqrt{t}) d r
\end{aligned}
$$

Or, equivalently,

$$
\frac{1}{h(\sqrt{t})} \int_{0}^{1} e^{-r^{2} / 4 t} \frac{r}{2 t} \mu\left(B_{r}(y) d r \leq \frac{1}{2} \int_{0}^{1 / \sqrt{t}} e^{-r^{2} / 4} r \frac{h(r \sqrt{t})}{h(\sqrt{t})} d r .\right.
$$

This integral is finite by Lemma 2.4.

For $r \geq 1$

$$
\begin{aligned}
\int_{1}^{\infty} e^{-r^{2} / 4 t} \frac{r}{2 t} \mu\left(B_{r}(y) d r\right. & \leq \int_{1}^{\infty} e^{-r^{2} / 4 t} \frac{r}{2 t} r^{n} d r \\
& =\frac{1}{2} \sqrt{t^{n}} \int_{1 / \sqrt{t}}^{\infty} e^{-r^{2} / 4} r^{n+1} d r
\end{aligned}
$$

Since $\lim _{t \rightarrow 0} t^{n} / h(t)=0$, we deduce that $\sqrt{t^{n}} / h(\sqrt{t}) \leq C$ and therefore

$$
\frac{1}{h(\sqrt{t})} \int_{1}^{\infty} e^{-r^{2} / 4 t} \frac{r}{2 t} \mu\left(B_{r}(y) d r \leq C,\right.
$$

with $C$ independent of $t$. This completes the second step of our proof.

Third (and last) step

We will now prove the thesis. Using the first and second steps we obtain:

$$
\begin{array}{r}
\frac{\sqrt{t^{n}}}{h(\sqrt{t})} \int\left|\mathcal{F}_{\mu} f(\xi)\right|^{2} e^{-t|\xi|^{2}} d \xi=\pi^{n / 2} \int\left(\int e^{-|x-y|^{2} / 4 t} f(x) d \mu(x)\right) f(y) d \mu(y) \\
\leq C \int M_{\mu} f(y)|f(y)| d \mu(y) .
\end{array}
$$


The last term is the inner product in the Hilbert space $L^{2}(\mu)$, then we can bound it using Cauchy-Schwartz. The $L^{2}(\mu)$ norm of $M_{\mu} f$ can be bounded by using the $(2,2)$ estimate in $(2.1)$. Then,

$$
\int M_{\mu} f(y)|f(y)| d \mu(y) \leq C\|f\|_{2}^{2}
$$

and this, together with (3.5) gives the desired result.

Theorem 3.2. Under the hypothesis of Theorem 3.1, for each $f \in L^{2}(\mu)$ we have:

$$
\sup _{x \in \mathbb{R}^{n}} \sup _{r \geq 1} \frac{1}{r^{n} h\left(r^{-1}\right)} \int_{B_{r}(x)}\left|\mathcal{F}_{\mu} f(\xi)\right|^{2} d \xi \leq C\|f\|_{2}^{2} .
$$

Proof. We need to show that for each $x \in \mathbb{R}^{n}$,

$$
\sup _{r \geq 1} \frac{1}{r^{n} h\left(r^{-1}\right)} \int_{B_{r}(x)}\left|\mathcal{F}_{\mu} f(\xi)\right|^{2} d \xi \leq C\|f\|_{2}^{2} .
$$

But making the substitution $t=r^{-2}$ in Theorem 3.1, we obtain, exactly (3.6) for $B_{r}(0)$. Further,

$$
\int_{B_{r}(x)}\left|\mathcal{F}_{\mu} f(\xi)\right|^{2} d \xi=\int_{B_{r}(0)}\left|\mathcal{F}_{\mu}\left(e^{i x \xi} f\right)\right|^{2} d \xi \leq C\left\|e^{i x \xi} f\right\|_{2}^{2}=C\|f\|_{2}^{2},
$$

which yields the Theorem.

This Theorem provides an upper bound but does not tell us weather the limit for $r \rightarrow \infty$ exists or not. With our definition, if a measure is $h$ dimensional it is also $g$ dimensional for any $h \leq g$. For example, if $h(x) \geq x^{n}$, then the measure $\mu=\mathcal{H}^{h}{ }_{\llcorner E}+\mathcal{L}$ (here, $\mathcal{L}$ is the $n$-dimensional Lebesgue measure and $E$ is a set of $\mathcal{H}^{h}$ finite measure) is an $h$-dimensional measure. However in this case it is clear that $\mu$ has two distinct parts, one 'truly' $h$-dimensional $\left(\mathcal{H}_{\llcorner E}^{h}\right)$ but the other $(\mathcal{L})$, even tough by the previous remark, can be considered as $h$-dimensional, is in fact $n$-dimensional.

The next Theorem will allow us to split up our measure in order to separate the part of the measure that is 'exactly' $h$-dimensional, from the one that can also be seen as having bigger dimension.

Definition 3.3. We say that a measure $\nu$ is null with respect to (another measure) $\mu$ if and only if,

$$
\mu(E)<\infty \Rightarrow \nu(E)=0 .
$$

We will denote this with $\nu \lll \mu$.

Now, we will prove a Theorem that is analogous to Radon Nikodym.

Theorem 3.4. Let $\mu$ a measure on $\mathbb{R}^{n}$ without infinitely many atoms and let $\nu$ be a $\sigma$-finite measure on $\mathbb{R}^{n}$ absolutely continuous with respect to $\mu$. There exists a unique decomposition of $\nu: \nu=\nu_{1}+\nu_{2}$, where $\nu_{1}(E)=\int_{E} f d \mu$ for some measurable and nonnegative function $f$, and $\nu_{2} \lll \mu$.

Proof. Uniqueness. Let us suppose we have a decomposition

$$
\nu=\nu_{1}+\nu_{2} \quad \text { with } \quad \nu_{1}(E)=\int_{E} f d \mu \quad \text { and } \quad \nu_{2} \lll \mu .
$$

Consider $E \subset \mathbb{R}^{n}$. Let us analyze separately both cases, when $E$ is $\sigma$-finite for $\mu$ and when it is not. 
If $E$ is $\sigma$-finite for $\mu$ then $E=\cup_{j \geq 1} E_{j}$ with $\mu\left(E_{j}\right)<\infty$. Since $\nu_{2} \lll \mu$ we have $\nu_{2}\left(E_{j}\right)=0$ for all $j \geq$ and therefore $\nu_{2}(E)=0$, which gives $\nu_{1}(E)=\nu(E)$. If we have any other decomposition $\nu=\nu_{1}^{\prime}+\nu_{2}^{\prime}$, then $\nu_{2}^{\prime}(E)=0=\nu_{2}(E)$ and $\nu_{1}^{\prime}(E)=\nu(E)=\nu_{1}(E)$.

If $E$ is not $\sigma$-finite for $\mu$, then $\nu_{2}$ may be positive. However, by hypothesis $\nu$ is still $\sigma$-finite and then $E=\cup_{j \geq 1} \tilde{E}_{j}$ with $\nu\left(\tilde{E}_{j}\right)<\infty\left(\tilde{E}_{j}\right.$ may be chosen disjoint if necessary). Suppose we have another decomposition $\nu=\nu_{1}^{\prime}+\nu_{2}^{\prime}$ with $\nu_{1}^{\prime}(E)=\int_{E} g d \mu$ and $\nu_{2}^{\prime} \lll \mu$. In particular, $\nu_{1}-\nu_{1}^{\prime}=\nu_{2}^{\prime}-\nu_{2}$. We have that

$$
\left(\nu_{1}-\nu_{1}^{\prime}\right)\left(\left\{x \in \tilde{E}_{j}: f(x)>g(x)\right\}\right)<\infty
$$

which by the definition of $\nu_{1}$ and $\nu_{1}$ ' implies that $\mu\left(\left\{x \in \tilde{E}_{j}: f(x)>g(x)\right\}\right)<\infty$. Since $\nu_{2}$ and $\nu_{2}^{\prime}$ are both null with respect to $\mu$ we have

$$
\nu_{2}\left(\left\{x \in \tilde{E}_{j}: f(x)>g(x)\right\}\right)=\nu_{2}^{\prime}\left(\left\{x \in \tilde{E}_{j}: f(x)>g(x)\right\}\right)=0 .
$$

We can do the same calculation for the complementary set for which $f(x)<g(x)$ and conclude that

$$
\nu_{2}\left(\tilde{E}_{j}^{\prime}\right):=\nu_{2}\left(\left\{x \in \tilde{E}_{j}: f(x) \neq g(x)\right\}\right)=\nu_{2}^{\prime}\left(\tilde{E}_{j}^{\prime}\right)=0
$$

and therefore,

$$
\nu_{1}\left(\tilde{E}_{j}^{\prime}\right)=\nu\left(\tilde{E}_{j}^{\prime}\right)=\nu_{1}^{\prime}\left(\tilde{E}_{j}^{\prime}\right) .
$$

In $\tilde{E}_{j} \backslash \tilde{E}_{j}^{\prime} f$ and $g$ coincide, and so $\nu_{1}\left(\tilde{E}_{j} \backslash \tilde{E}_{j}^{\prime}\right)=\nu_{1}\left(\tilde{E}_{j} \backslash \tilde{E}_{j}^{\prime}\right)$. Since $\tilde{E}_{j}=$ $\tilde{E}_{j}^{\prime} \cup\left(\tilde{E}_{j} \backslash \tilde{E}_{j}^{\prime}\right)$ it follows that $\nu_{1}$ and $\nu_{1}^{\prime}$ coincide on each $\tilde{E}_{j}$, and therefore on $E$, if the $\tilde{E}_{j}$ were chosen disjoint.

Now it follows that $\nu_{2}=\nu_{2}^{\prime}$.

Existence. Let consider -first- the case when $\nu$ is finite. We define the set

$$
\mathscr{A}=\left\{A \subset \mathbb{R}^{n}: A \text { is measurable, } \nu(A)>0, \mu_{\llcorner A} \text { is } \sigma-\text { finite }\right\} .
$$

If $\mathscr{A}=\emptyset$, then the theorem follows taking $\nu_{2}=\nu$ and $\nu_{1}=0$. If $\mathscr{A} \neq \emptyset$, define $a:=\sup _{A \in \mathscr{A}} \nu(A)$. We have that $a$ is finite, since $\nu$ is. Consider the set sequence $\left(A_{j}\right)_{j \in \mathbb{N}} \subset \mathscr{A}$ such that $\nu\left(A_{j}\right) \rightarrow a$. Let $B:=\bigcup_{j=1}^{\infty} A_{j}$. We are going to see that we can take $\nu_{1}=\nu_{\llcorner B}$ and $\nu_{2}=\nu_{\left\llcorner B^{c}\right.}$. In fact, since $\mu_{\llcorner B}$ is $\sigma$-finite, we have $f$, the Radon Nykodim derivative of $\nu$ with respect to $\mu\left\llcorner_{B}\right.$. Now, we take a set $E$ such that $\mu(E)<\infty$. If $\nu_{2}(E)>0$, then $\nu(E \cup B)>a$ which is a contradiction. Therefore $\nu_{2}(E)=0$, and so, $\nu_{2} \lll \mu$.

Let analyze now the case when $\nu$ is not finte (but still $\sigma$ finite). Let $\left(E_{j}\right)$ be a collection of measurable sets with $\nu\left(E_{j}\right)<\infty$ such that $\cup E_{j}=E$. Without loss of generality, we can asume that $E_{j}$ are disjoint. We define $\nu^{j}=\nu\left\llcorner E_{j}\right.$ y $\mu^{j}=\nu\left\llcorner E_{j}\right.$. Then $\nu^{j}$ is finite and regarding the previous case we can decompose $\nu^{j}=\nu_{1}^{j}+\nu_{2}^{j}$. Now, $\nu_{1}=\sum_{j} \nu_{1}^{j}$ and $\nu_{2}=\sum_{j} \nu_{2}^{j}$ verify the thesis.

Corollary 3.5. If $\mu$ is an $h$-dimensional measure, then there exists $\varphi \geq 0$ and $\nu \lll \mathcal{H}^{h}$ such that $\mu=\varphi d \mathcal{H}^{h}+\nu$.

Proof. In view of the previous Theorem, we only need to prove that $\mu$ is absolutely continuous respect to $\mathcal{H}^{h}$. Let us take a set $E$ with $\mathcal{H}^{h}(E)=0$. Then, for any $\varepsilon>0$, there is a cover $\left(U_{i}\right)_{i \geq 1}$ of $E$ with $\sum_{i=1}^{\infty} h\left(\left|U_{i}\right|\right)<\varepsilon$, where $\left|U_{i}\right|$ is the diameter of $U_{i}$. Then,

$$
\mu(E) \leq \sum_{i=1}^{\infty} \mu\left(U_{i}\right) \leq \sum_{i=1}^{\infty} \mu\left(B_{\left|U_{i}\right|}\left(x_{i}\right)\right)
$$


picking any $x_{i} \in U_{i}$. Now, using that $\mu$ is $h$-dimensional and the previous estimate, we have,

$$
\mu(E) \leq \sum_{i=1}^{\infty} h\left(\left|U_{i}\right|\right)<\varepsilon .
$$

Since $\varepsilon$ is arbitrary $\mu(E)=0$ and the proof is complete.

The next technical lemma will be necessary for our construction.

Lemma 3.6. If $\nu$ is a locally finite measure on $\mathbb{R}^{n}$ and $\nu \lll \mathcal{H}^{h}$, then $\bar{D}_{h}(\nu, x):=$ $\limsup _{r \rightarrow 0} \frac{\nu\left(B_{r}(x)\right)}{h(2 r)}=0$ for $\mathcal{H}^{h}$ - almost every $x$.

Proof. For each $k \in \mathbb{N}$, we define the sets

$$
E_{k}=\left\{x \in \mathbb{R}^{n}: \forall \varepsilon>0 \quad \exists r \leq \varepsilon \text { with } \frac{\nu\left(B_{r}(x)\right)}{h(2 r)} \geq \frac{1}{k}\right\}
$$

Since,

$$
\left\{x \in \mathbb{R}^{n}: \bar{D}_{h}(\nu, x)>0\right\}=\bigcup_{k \geq 1} E_{k},
$$

it is enough to prove that $\mathcal{H}^{h}\left(E_{k}\right)=0$ for all $k$.

We can suppose that $\nu\left(E_{k}\right)$ is finite, since $E_{k}=\cup_{l \geq 1}\left(E_{k} \cap B_{l}(0)\right)$.

Let $k$ be fixed and let $\varepsilon>0$. For each $x \in E_{k}$, we can pick an $r(x) \leq \varepsilon$ such that $h(2 r(x)) \leq k \nu\left(B_{r(x)}(x)\right) . \quad\left\{B_{r(x)}(x)\right\}_{x \in E_{k}}$ is a family of balls with uniformly bounded radii. Therefore, by Besicovitch's covering Theorem ([5]) we can take a countable subcover $\left\{B_{r_{j}}\left(x_{j}\right)\right\}_{j \geq 1}$ of $E_{k}$ such that at most $c(n)$ of the balls intersect at once (i.e. $\left.\sum \chi_{B_{r_{j}}} \leq c(n)\right)$.

Now, since $r_{j} \leq \varepsilon$, it follows that $B_{r_{j}} \subset E_{k, \varepsilon}:=\left\{x \in \mathbb{R}^{n}: \operatorname{dist}\left(x, E_{k}\right) \leq \varepsilon\right\}$. So we have:

$$
\sum_{j=1}^{\infty} h\left(2 r_{j}\right) \leq k \sum_{j=1}^{\infty} \nu\left(B_{r_{j}}\left(x_{j}\right)\right) \leq k c(n) \nu\left(E_{k, \varepsilon}\right),
$$

and therefore, $\mathcal{H}^{h}\left(E_{k}\right) \leq c(n) k \nu\left(E_{k, \varepsilon}\right)$.

But since $E_{k} \subset \cap_{\varepsilon>0} E_{k, \varepsilon}$ and $\nu\left(E_{k}\right)$ is finite, we have that $\mathcal{H}^{h}\left(E_{k}\right) \leq c(n) k \nu\left(E_{k}\right)$. In particular, $\mathcal{H}^{h}\left(E_{k}\right)$ is finite, which implies $\nu\left(E_{k}\right)=0$ by the hypothesis on $\nu$.

Using again that $\mathcal{H}^{h}\left(E_{k}\right) \leq c(n) k \nu\left(E_{k}\right)$, we obtain the desired result.

We are now able to establish a finer bound for certain $h$-dimensional measures (compare with Theorem 3.1 and Theorem 3.2).

Theorem 3.7. Let $\mu$ be any $h$ dimensional measure and let $\mu=\varphi d \mathcal{H}^{h}+\nu$ (with $\left.\nu \lll \mathcal{H}^{h}\right)$ be the decomposition of Theorem 3.4. If $f \in L^{2}(\mu)$ then

$$
\limsup _{t \rightarrow 0} \frac{\sqrt{t^{n}}}{h(\sqrt{t})} \int e^{-t|\xi|^{2}}\left|\mathcal{F}_{\mu} f(\xi)\right|^{2} d \xi \leq c \int|f(x)|^{2} \varphi(x) d \mathcal{H}^{h}(x)
$$

and

$$
\sup _{y \in \mathbb{R}^{n}} \limsup _{r \rightarrow \infty} \int_{B_{r}(y)}\left|\mathcal{F}_{\mu} f(\xi)\right|^{2} d \xi \leq c \int|f(x)|^{2} \varphi(x) d \mathcal{H}^{h}(x)
$$


Proof. For this proof we will use the maximal operator $M_{\mu}$ as defined in (2.1). It suffices to prove that

$$
\lim _{t \rightarrow 0} \frac{\sqrt{t^{n}}}{h(\sqrt{t})} \int e^{-t|\xi|^{2}}\left|\mathcal{F}_{\nu} f(\xi)\right|^{2} d \xi=0
$$

and

$$
\lim _{t \rightarrow 0} \frac{\sqrt{t^{n}}}{h(\sqrt{t})} \int e^{-t|\xi|^{2}} \mathcal{F}_{\nu} f(\xi) \quad \mathcal{F}_{\mathcal{H}^{n}} f(\xi) \quad d \xi=0 .
$$

Doing the same type of computations than the ones used to obtain (3.4), we have:

$$
\frac{1}{h(\sqrt{t})} \int e^{-|x-y|^{2} / 4 t} f(x) d \nu(x) \leq \frac{M_{\nu} f(y)}{h(\sqrt{t})} \int_{0}^{\infty} e^{-r^{2} / 4 t} \frac{r}{2 t} \nu\left(B_{r}(y)\right) d r .
$$

On the other hand, by Lemma 3.6, for $\mathcal{H}^{h}$-almost every $y$,

$$
\bar{D}_{h}(\nu, y)=\limsup \frac{\nu\left(B_{r}(y)\right)}{h(2 r)}=0
$$

and therefore for all $\varepsilon>0$ we can choose $0<\delta<1$ such that $\nu\left(B_{r}(y)\right) \leq \varepsilon h(r)$.

We split the integral on the right of (3.9) into two parts: $\int_{0}^{\delta}+\int_{\delta}^{\infty}$. For the first one, using that $\nu\left(B_{r}(x)\right) \leq \mu\left(B_{r}(x)\right)$, and so $h$-dimensional, we obtain:

$$
\begin{array}{r}
\frac{M_{\nu} f(y)}{h(\sqrt{t})} \int_{0}^{\delta} e^{-r^{2} / 4 t} \frac{r}{2 t} \nu\left(B_{r}(x)\right) d r \leq M_{\nu} f(y) \varepsilon \int_{0}^{\delta / \sqrt{t}} e^{-r^{2} / 4} r \frac{h(r \sqrt{t})}{h(\sqrt{t})} d r \\
\leq c \varepsilon M_{\nu} f(y),
\end{array}
$$

by hypothesis.

For the second one, we split again:

$$
\begin{aligned}
& \frac{M_{\nu} f(y)}{h(\sqrt{t})} \int_{\delta}^{\infty} e^{-r^{2} / 4 t} \frac{r}{2 t} \nu\left(B_{r}(y)\right) d r \\
& \quad \leq M_{\nu} f(y) c\left(\int_{\delta / \sqrt{t}}^{1 / \sqrt{t}} e^{-r^{2} / 4} r \frac{h(r \sqrt{t})}{h(\sqrt{t})} d r+\int_{1 / \sqrt{t}}^{\infty} e^{-r^{2} / 4} r^{n+1} \frac{\sqrt{t^{n}}}{h(\sqrt{t})} d r\right) \rightarrow{ }_{t \rightarrow 0} 0 .
\end{aligned}
$$

So, if we denote by

$$
H(t, y):=\frac{1}{h(\sqrt{t})} \int e^{-|x-y|^{2} / 4 t} f(x) d \nu(x),
$$

we showed that $\lim _{t \rightarrow 0} H(t, y)=0$. Using dominated convergence in the same way than it was used in the first step of the proof of Theorem 3.1, we obtain,

$$
\begin{aligned}
0=\int \lim _{t \rightarrow 0} H(t, y) \overline{f(y)} d \nu(y) & =\lim _{t \rightarrow 0} \frac{1}{h(\sqrt{t})} \int e^{-|x-y|^{2} / 4 t} f(x) d \nu(x) \overline{f(y)} d \nu(y) \\
& =\lim _{t \rightarrow 0} \frac{\sqrt{t^{n}}}{h(\sqrt{t})} \int e^{-t|\xi|^{2}}\left|\mathcal{F}_{\nu} f(\xi)\right|^{2} d \xi
\end{aligned}
$$

In the same way, if we integrate with respect to $\mu$, we obtain

$$
\lim _{t \rightarrow 0} \frac{\sqrt{t^{n}}}{h(\sqrt{t})} \int e^{-t|\xi|^{2}} \mathcal{F}_{\nu} f(\xi) \mathcal{F}_{\mu} f(\xi) d \xi=0 .
$$

Now, the thesis is a consequence of Theorems 3.1 and 3.2 


\section{LOWER ESTimate.}

In this section we estimate the lower bound for the $\mu$-Fourier transform. We start by the following theorem.

Theorem 4.1. Let $\mu=\mathcal{H}^{h_{\left\llcorner_{E}\right.}}$ for an h-regular set $E$ (see 1.2).

Suppose that the function $h$ satisfies that:

$$
h(t) \leq t^{n} \text { for } t \geq 1 \text { and } \lim _{t \rightarrow 0} \frac{t^{n}}{h(t)}=0 .
$$

Also, suppose that the limit:

$$
\lim _{t \rightarrow 0} \frac{h(r t)}{h(t)}:=p(r)
$$

exists. Then, for $f \in L^{2}(\mu)$,

$$
\lim _{t \rightarrow 0} \frac{\sqrt{t^{n}}}{h(\sqrt{t})} \int e^{-t|\xi|^{2}}\left|\mathcal{F}_{\mu} f(\xi)\right|^{2} d \xi=C_{n, h} \int|f|^{2} d \mu
$$

where $C_{n, h}=\int_{0}^{\infty} e^{-r^{2} / 2} r p(r) d r$.

Proof. In view of (3.2), we will estimate

$$
\frac{\sqrt{t^{n}}}{h(\sqrt{t})} \int_{0}^{\infty} e^{-r^{2} / 4 t} \frac{r}{2 t} \int_{B_{r}(y)} f(x) d \mu(x) d r .
$$

We write the first integral as sum of: $\int_{0}^{\delta}+\int_{\delta}^{\infty}$. For any $\delta$ the second one tends to zero, since:

$$
\begin{aligned}
& \frac{1}{h(\sqrt{t})} \int_{\delta}^{\infty} e^{-r^{2} / 4 t} \frac{r}{2 t} \int_{B_{r}(y)} f(x) d \mu(x) d r \\
& \quad \leq \frac{1}{h(\sqrt{t})} \int_{\delta}^{\infty} e^{-r^{2} / 4 t} \frac{r}{2 t} \mu\left(B_{r}(y)\right) M_{\mu} f(y) d r \\
& \quad \leq \frac{1}{h(\sqrt{t})} M_{\mu} f(y)\left(\int_{\delta}^{1} e^{-r^{2} / 4 t} \frac{r}{2 t} h(r) d r+\int_{1}^{\infty} e^{-r^{2} / 4 t} \frac{r}{2 t} r^{n} d r\right) \\
& \quad=\frac{\sqrt{t^{n}}}{h(\sqrt{t})} M_{\mu} f(y)\left(\int_{\delta / \sqrt{t}}^{1 / \sqrt{t}} r h(r) e^{-r^{2} / 4} d r+\int_{1 / \sqrt{t}}^{\infty} r^{n+1} e^{-r^{2} / 4} d r\right) \underset{t \rightarrow 0}{\longrightarrow} 0
\end{aligned}
$$

using that $\lim _{t \rightarrow 0} \frac{t^{n}}{h(t)}=0$.

To analyze the other integral, note first that since $E$ is regular by Corollary 2.3 we have that, for $\mathcal{H}^{h}$-almost every $y \in E$ (fixed), and for all $\varepsilon>0$ there exists $\delta>0$ such that

$$
\left|\int_{B_{r}(y)} f d \mu-h(r) f(y)\right| \leq \varepsilon h(r) \quad \forall r \leq \delta .
$$

On the other hand,

$$
\int_{0}^{\delta} \frac{1}{h(\sqrt{t})} e^{-r^{2} / 4 t} \frac{r}{2 t} h(r) f(y) d r=f(y) \int_{0}^{2 \delta / \sqrt{t}} e^{-r^{2}} r \frac{h(r \sqrt{t})}{h(\sqrt{t})} d r
$$


and so, since $e^{-r^{2}} r \frac{h(r \sqrt{t})}{h(\sqrt{t})}$ is dominated by $e^{-r^{2}} r^{1+\kappa}$ (see Lemma 2.4), we have that

$$
\int_{0}^{\delta} \frac{1}{h(\sqrt{t})} e^{-r^{2} / 4 t} \frac{r}{2 t} h(r) f(y) d r \underset{t \rightarrow 0}{\longrightarrow} f(y) \int_{0}^{\infty} e^{-r^{2}} r p(r) d r .
$$

We conclude that

$$
\int_{0}^{\delta} \frac{1}{h(\sqrt{t})} e^{-r^{2} / 4 t} \frac{r}{2 t} \int_{B_{r}(y)} f(x) d \mu(x) d r \underset{t \rightarrow 0}{\longrightarrow} C_{n, h} f(y) .
$$

Combining (4.2) and (4.4) we obtain that

$$
H(t, y):=\frac{1}{h(\sqrt{t})} \int_{0}^{\infty} e^{-r^{2} / 4 t} \frac{r}{2 t} \int_{B_{r}(y)} f d \mu d r \underset{t \rightarrow 0}{\longrightarrow} C_{n, h} f(y) .
$$

Since $H(t, y)$ is dominated by $f(y) \int_{0}^{\infty} e^{-r^{2}} r p(r) d r$ and $f \in L^{2}(\mu)$, it follows that

$$
\lim _{t \rightarrow 0} \int H(t, y) \overline{f(y)} d \mu(y)=\int \lim _{t \rightarrow 0} H(t, y) \overline{f(y)} d \mu(y)=C_{n, h} \int_{E}|f|^{2} d \mu .
$$

Note that equation (4.3), which was very important in our proof is a reformulation of Corollary 2.2 substituting $\mu\left(B_{r}(y)\right)$ by $h(r)$. We are allowed to make this substitution only because $E$ is a regular set. However, this hypothesis on $E$ is too restrictive.

Actually, it has already been proven (see [5]) that there only exist regular sets, for functions of the form $x^{k}$ with $k$ integer. So, in order for the last Theorem to be meaningful, it will be necessary to obtain a result with weaker hypothesis. We will therefore consider $h$-quasi regular sets, meaning that there exists a constant $\theta>0$ such that for $\mathcal{H}^{h}$ almost every $x \in E$,

$$
\liminf _{r \rightarrow 0} \frac{\mathcal{H}^{h}\left(B_{r}(x) \cap E\right)}{h(r)} \geq \theta
$$

For this case, instead of the equality in (4.1) we obtain a lower bound.

Theorem 4.2. Let $\mu=\mathcal{H}_{E}^{h}+\nu$. If $\nu \lll \mathcal{H}^{h}$ and $E$ is h-quasi regular, we have:

$$
\liminf _{t \rightarrow 0} \int e^{-t|\xi|^{2}}\left|\mathcal{F}_{\mu} f(\xi)\right|^{2} d \xi \geq c \int_{E}|f|^{2} d \mathcal{H}^{h}
$$

Proof. By the proof of Theorem 3.7, we can suppose $\mu=\mathcal{H}^{h}\left\llcorner_{E}\right.$.

Since $E$ is quasi regular there exists $\delta_{1}>0$ such that if $r<\delta_{1}$ then,

$$
\mu\left(B_{r}(x)\right) \geq \operatorname{ch}(r) .
$$

On the other hand, there exists $\delta_{2}>0$ such that if $r<\delta_{2}$ (and $f(y) \neq 0$ ) then,

$$
\left|\frac{1}{\mu\left(B_{r}(y)\right)} \int_{B_{r}(y)} f(x) d \mu(x)-f(y)\right|<\varepsilon|f(y)| .
$$

Taking $\delta=\delta_{y, \varepsilon}$ satisfying both estimates, we may write

$$
\frac{1}{h(\sqrt{t})} \iint e^{-|x-y|^{2} / 4 t} f(x) \overline{f(y)} d \mu(x) d \mu(y)=\int|f(y)|^{2} H(y, t, \varepsilon) d \mu(y)+R(t, \varepsilon),
$$


where $H(y, t, \varepsilon)=\int_{0}^{\delta_{y, \varepsilon}} \frac{1}{h(\sqrt{t})} e^{-r^{2} / 4 t} \frac{r}{2 t} \mu\left(B_{r}(y)\right) d r$ and

$$
\begin{aligned}
R(t, \varepsilon)=\int \overline{f(y)} \int_{0}^{\delta_{y, \varepsilon}} & \frac{e^{-r^{2} / 4 t}}{h(\sqrt{t})} \frac{r}{2 t}\left(\int_{B_{r}(y)} f(x) d \mu(x)-f(y) \mu\left(B_{r}(y)\right)\right) d r d \mu(y) \\
& +\int \overline{f(y)} \int_{\delta_{\varepsilon, y}}^{\infty} \frac{1}{h(\sqrt{t})} e^{-r^{2} / 4 t} \frac{r}{2 t} \int_{B_{r}(y)} f(x) d \mu(x) d r d \mu(y) .
\end{aligned}
$$

We are now going to bound $|R(t, \varepsilon)|$. Using (4.9) and the fact that there exist a bound independent of $t$ for $\int_{0}^{\delta} \frac{e^{-r^{2} / 4 t}}{h(\sqrt{t})} \frac{r}{2 t} \mu\left(B_{r}(y)\right) d r$ we can bound the first term by $C_{1} \varepsilon\|f\|^{2}$. The second one is bounded by

$$
\int|f(y)| \int_{\delta_{\varepsilon, y}}^{\infty} \frac{1}{h(\sqrt{t})} e^{-r^{2} / 4 t} \frac{r}{2 t} \mu\left(B_{r}(y)\right) d r M_{\mu} f(y) d \mu(y),
$$

and remembering a previous calculation, the integral $\int_{\delta}^{\infty} \frac{e^{-r^{2} / 4 t}}{h(\sqrt{t})} \frac{r}{2 t} \mu\left(B_{r}(y)\right) d r$ can be bounded by $\varepsilon$ if we take $t$ small enough. Therefore, by Cauchy Schwartz and the $(2,2)$ hard estimate, the second term is bounded by $C_{2}\|f\|^{2}$. So, both estimates tell us that $|R(t, \varepsilon)| \leq C\|f\|^{2}$ for small enough $t$.

On the other hand, $H(y, t, \varepsilon)$ is bounded bellow by $\int_{0}^{\delta} \frac{e^{-r^{2} / 4 t}}{h(\sqrt{t})} \frac{r}{2 t} h(r) d r$, using (4.8). Substituting and using that $\liminf _{t \rightarrow 0} \frac{h(r \sqrt{t})}{h(\sqrt{t}}<\infty$ we conclude that $\liminf _{t \rightarrow 0} H(y, t, \varepsilon) \geq C_{3}$.

Therefore by Fatou's Lemma

$$
\liminf _{t \rightarrow 0} \frac{1}{h(\sqrt{t})} \iint e^{-|x-y|^{2} / 4 t} f(x) f(y) d \mu(x) d \mu(y) \geq c \int|f|^{2} d \mu .
$$

Theorem 4.3. Let $\mu$ an $h$-dimensional measure such that $\mu=\mathcal{H}^{h}\left\llcorner_{E}+\nu\right.$ with $\nu \lll \mathcal{H}^{h}$ being $E$ h-quasi regular. Then, the following inequality holds:

$$
\liminf _{r \rightarrow \infty} \frac{1}{r^{n} h\left(r^{-1}\right)} \int_{B_{r}(y)}\left|\mathcal{F}_{\mu} f(\xi)\right|^{2} d \xi \geq c \int_{E}|f|^{2} d \mathcal{H}^{h},
$$

where the constant $c$ does not depend on $y$.

Proof. For any $\lambda>0$ such that $\lambda \leq t|\xi|^{2}$ we have $e^{-t|\xi|^{2}} \leq e^{-\lambda / 2} e^{-(1 / 2) t|\xi|^{2}}$. Then,

$$
\begin{array}{cl}
\frac{\sqrt{t}^{n}}{h(\sqrt{t})} \int_{\left\{\xi: t|\xi|^{2} \geq \lambda\right\}} & e^{-t|\xi|^{2}}\left|\mathcal{F}_{\mu} f(\xi)\right|^{2} d \xi \\
\leq & 2^{n / 2} \frac{h\left((t / 2)^{1 / 2}\right)}{h(\sqrt{t})} \frac{(t / 2)^{n / 2}}{h\left((t / 2)^{1 / 2}\right)} e^{-\lambda / 2} \int e^{-(1 / 2) t|\xi|^{2}}\left|\mathcal{F}_{\mu}(\xi)\right|^{2} d \xi \\
\leq & c e^{-\lambda / 2} \int_{E}|f|^{2} d \mathcal{H}^{h}
\end{array}
$$

by Lemma 2.4 and Theorem 3.7. Using 4.7 and picking $\lambda$ big enough, we obtain:

$$
\liminf _{t \rightarrow 0} \frac{\sqrt{t^{n}}}{h(\sqrt{t})} \int_{\left\{\xi: t|\xi|^{2} \leq \lambda\right\}} e^{-t|\xi|^{2}}\left|\mathcal{F}_{\mu}(\xi)\right|^{2} d \xi \geq \tilde{c} \int_{E}|f|^{2} d \mu
$$


picking the constant $c$ smaller if it is needed. Now, taking $t=\lambda / r^{2}$, we obtain

$$
\frac{h\left(\lambda^{1 / 2}\right)}{\lambda^{n / 2}} \frac{\sqrt{t^{n}}}{h(\sqrt{t})} \int_{\left\{\xi: t|\xi|^{2} \leq \lambda\right\}} e^{-t|\xi|^{2}}\left|\mathcal{F}_{\mu}(\xi)\right|^{2} d \xi \leq c_{\lambda} \frac{1}{r^{n} h\left(r^{-1}\right)} \int_{B_{r}(0)}\left|\mathcal{F}_{\mu}(\xi)\right|^{2} d \xi,
$$

where $c_{\lambda}$ is such that $h\left(r^{-1}\right) / h\left(\lambda^{1 / 2} r^{-1}\right) \leq c_{\lambda}$. This completes the proof.

\section{An example}

We conclude the paper by exhibiting an example of a function $h$ and a set $C$ such that $\mathcal{H}_{\llcorner C}^{h}$ is $h$-dimensional and $C$ is quasi regular. For this example Theorem 4.3 holds. However, since $C$ is $\alpha$ dimensional but with zero $\mathcal{H}^{\alpha}$ measure the results of Strichartz in [10] do not apply. This shows that by considering more general dimension functions we obtained a useful generalization.

Let $h:[0, \infty) \rightarrow \mathbb{R}$ be a dimension function such that $h(2 x)<2 h(x)$. Let $s_{k}$ be such that $h\left(s_{k}\right)=2^{-k}$. We will construct a set of Cantor type. Consider the two (closed) subintervals of $[0,1] I_{1,1}$ and $I_{1,2}$, of length $s_{1}$ obtained by suppressing the central open interval of length $1-2 s_{1}$. In each of these intervals we take the two closed subinterval of length $s_{2}$ obtained by removing the central interval of length $s_{1}-2 s_{2}$ this time (note that this number is positive because $h(2 x)<2 h(x)$ ). We obtain four intervals denoted by $I_{2,1}, I_{2,2}, I_{2,3}, I_{2,4}$. These intervals will be called intervals of step 2. Following in the same manner at each step, we obtain $2^{k}$ closed intervals of length $s_{k}$. Our Cantor set will be:

$$
C=\bigcap_{k \geq 1} \bigcup_{j=1}^{2^{k}} I_{k, j} .
$$

We assign to each interval $I_{k, j}$ measure $2^{-k}$ obtaining a probability measure $\mu$ supported on $C$. We can see ([3]) that this measure is $\mathcal{H}^{h}\llcorner C$.

We are going to show that this set satisfies the hypothesis of the Theorem 4.2, what means, essentially, that it is $h$-quasi regular. It suffices to see that $\frac{\mu(B(x, \rho))}{h(2 \rho)} \geq c$ (where $c$ is a positive constant) for all $x \in C$ and for all $\rho>0$.

Given $x \in C$ and $\rho>0$, denote by $k$ the minimum integer such that there exists $j$ between 1 and $2^{k}$ satisfying $I_{k, j} \subset B(x, \rho)$. By minimality $s_{k-1} \geq \rho$. Then,

$$
\frac{\mu(B(x, \rho))}{h(2 \rho)} \geq \frac{\mu\left(I_{k, j}\right)}{h(2 \rho)}=\frac{2^{-k}}{h(2 \rho)} \geq \frac{c_{d}}{2} \frac{1}{2^{k-1} h(\rho)} \geq \frac{c_{d}}{2} \frac{1}{2^{k-1} h\left(s_{k-1}\right)}=\frac{c_{d}}{2},
$$

using that $I_{k, j} \subset B(x, \rho)$, the definition of $\mu$, the Lemma 2.4, the minimality of $k$ and the definition of $s_{k}$. Therefore (4.6) follows.

We also need to prove that $\mu=\mathcal{H}^{h}\left\llcorner_{C}\right.$ is an $h$ - dimensional measure. In fact, $C \cap B_{\rho}(x) \subset I_{k-1, j}$ for some $j$. Consequently

$$
\mu\left(B_{\rho}(x)\right) \leq \mu\left(I_{k-1, j}\right)=2^{-(k-1)}=2 h\left(s_{k}\right) \leq h(\rho) .
$$

If we take $h(x)=x^{\alpha} \log (1 / x)$, then we obtain a set $C$ of dimension $\alpha$ but such that $\mathcal{H}^{\alpha}(C)=0$. Therefore for any $\alpha, C$ will not be $\alpha$-quasi regular, and hence we can not apply Strichartz's Theorem.

However, since $C$ is $h$-quasi regular for $h(x)=x^{\alpha} \log (1 / x)$, we can apply Theorem 4.3 


\section{REFERENCES}

[1] John J. Benedetto and Joseph D. Lakey. The definition of the Fourier transform for weighted inequalities. J. Funct. Anal., 120(2):403-439, 1994.

[2] C. Cabrelli, F. Mendivil, U. Molter, and R. Shonkwiler. On the $h$-hausdorff measure of cantor sets. Pacific Journal of Mathematics, 217(1):29-43, 2004.

[3] K. J. Falconer. The geometry of fractal sets. Cambridge University Press, Cambridge, 1985.

[4] K. J. Falconer. Techniques in Fractal Geometry. John Wiley \& Sons, New York, 1997.

[5] P. Mattila. Geometry of Sets and Measures in Euclidean Spaces. Cambridge University Press, Cambridge, 1995.

[6] C. A. Rogers. Hausdorff Measures. Cambridge University Press, Cambridge, UK, second edition, 1998.

[7] R. Strichartz. Self-similar measures and their Fourier Transforms I. Indiana University Mathematics Journal, 39(3):797-817, 1990.

[8] R. Strichartz. Self similar measures and their fourier transform II. Trans. Amer. Math. Soc., 1993.

[9] R. Strichartz. Self-similarity in Harmonic Analysis. The Journal of Fourier Analysis and Applications, 1(1):1-37, 1994.

[10] R. Stricharz. Fourier asymptotics of fractal measures. Journal of Functional Analysis, 89:154$187,1990$.

Depto. de Matemática, FCEyn, Univ. de Buenos Aires, Cdad. Univ., Pab. I, 1428 Capital Federal, argentina

E-mail address: umolter@dm.uba.ar

E-mail address: zuberman@dm.uba.ar 